\title{
RESEARCH
}

\section{Effect of mechanical ventilation in the prone position on clinical outcomes in patients with acute hypoxemic respiratory failure: a systematic review and meta-analysis}

\author{
Sachin Sud MD, Maneesh Sud BSc, Jan O. Friedrich MD DPhil, Neill K.J. Adhikari MDCM MSc
}

$\infty$

See related article page $\mathrm{II} 74$

\section{ABSTRACT}

Background: Mechanical ventilation in the prone position is used to improve oxygenation in patients with acute hypoxemic respiratory failure. We sought to determine the effect of mechanical ventilation in the prone position on mortality, oxygenation, duration of ventilation and adverse events in patients with acute hypoxemic respiratory failure.

Methods: In this systematic review we searched MEDLINE, EMBASE, the Cochrane Central Register of Controlled Trials and Science Citation Index Expanded for articles published from database inception to February 2008. We also conducted extensive manual searches and contacted experts. We extracted physiologic data and clinically relevant outcomes.

Results: Thirteen trials that enrolled a total of 1559 patients met our inclusion criteria. Overall methodologic quality was good. In 10 of the trials $(n=1486)$ reporting this outcome, we found that prone positioning did not reduce mortality among hypoxemic patients (risk ratio [RR] 0.96, 95\% confidence interval $[\mathrm{Cl}] 0.84^{-1.09} ; p=0.52$ ). The lack of effect of ventilation in the prone position on mortality was similar in trials of prolonged prone positioning and in patients with acute lung injury. In 8 of the trials $(n=633)$, the ratio of partial pressure of oxygen to inspired fraction of oxygen on day 1 was $34 \%$ higher among patients in the prone position than among those who remained supine $(p<0.001)$; these results were similar in 4 trials on day 2 and in 5 trials on day $3 . \ln 9$ trials $(n=1206)$, the ratio in patients assigned to the prone group remained $6 \%$ higher the morning after they returned to the supine position compared with patients assigned to the supine group $(p=0.07)$. Results were quantitatively similar but statistically significant in 7 trials on day 2 and in 6 trials on day $3(p=0.001)$. In 5 trials $(n=1004)$, prone positioning was associated with a reduced risk of ventilator-associated pneumonia (RR $0.81,95 \% \mathrm{CI}$ $0.66-0.99 ; p=0.04$ ) but not with a reduced duration of ventilation. In 6 trials $(n=504)$, prone positioning was associated with an increased risk of pressure ulcers (RR 1.36 , $95 \% \mathrm{Cl} 1.07-1.71 ; p=0.01)$. Most analyses found no to moderate between-trial heterogeneity.
Interpretation: Mechanical ventilation in the prone position does not reduce mortality or duration of ventilation despite improved oxygenation and a decreased risk of pneumonia. Therefore, it should not be used routinely for acute hypoxemic respiratory failure. However, a sustained improvement in oxygenation may support the use of prone positioning in patients with very severe hypoxemia, who have not been well-studied to date.

Une version française de ce résumé est disponible à l'adresse www.cmaj.ca/cgi/content/full/I78/9/II53/DCI

CMAJ 2008;178(9):1153-6I

$\mathrm{P}$ atients with acute lung injury ${ }^{1,2}$ and hypoxemic respiratory failure may require mechanical ventilation to maintain oxygenation. Persistent hypoxemia may entail additional treatments, such as inhaled nitric oxide ${ }^{3}$ or high-frequency oscillation, ${ }^{4-6}$ but these treatments are not universally available. In contrast, ventilation in the prone position, first recommended in $1974,{ }^{7}$ can be readily implemented in any intensive care unit (ICU), and clinicians should be familiar with its effects on patient outcomes.

Improved ventilation-perfusion matching is the major physiologic effect of prone positioning for ventilation in patients with acute lung injury. ${ }^{8}$ In the supine position, the dependent dorsal lung regions (compared with nondependent regions) are atelectatic owing to decreased transpulmonary pressure and direct compression by the lungs, heart and abdominal contents (via pressure on a passive diaphragm). Gravity favours increased perfusion to these collapsed dorsal lung segments, which creates shunt conditions. In the prone position, lung compression is decreased, and chest-wall and lung mechanics create more uniform transpulmonary pressure. The previously atelectatic lung thus becomes aerated,

From the Interdepartmental Division of Critical Care (S. Sud, Friedrich, Adhikari), University of Toronto; the Faculty of Science (M. Sud [at the time of writing]), University of Toronto, Toronto, Ont. (current affiliation: Faculty of Medicine, University of Manitoba, Winnipeg, Man.); the Departments of Critical Care and Medicine and the Li Ka Shing Knowledge Institute (Friedrich), St. Michael's Hospital, Toronto, Ont.; and the Department of Critical Care Medicine (Adhikari), Sunnybrook Health Sciences Centre, Toronto, Ont. 
and new atelectasis in the now dependent ventral regions is comparatively minor. In addition, lung perfusion in the prone position is more homogeneous. Shunt conditions are therefore reduced and ventilation is better matched to perfusion. Other clinical effects of prone positioning may include enhanced postural drainage of secretions, ${ }^{9,10}$ decreasing the risk of ventilator-associated pneumonia. Effects may also include decreased alveolar overdistension, cyclic alveolar collapse and ventilator-induced lung injury. ${ }^{11}$ For this reason, some investigators have recommended prone positioning for mechanical ventilation in the treatment of acute lung injury. ${ }^{8,11}$

Although ventilation in the prone position offers physiologic advantages and does not require specialized tools, one survey found that in most ICUs, 3 personnel (range 2-6) were required to turn an adult patient. ${ }^{12}$ These caregivers must handle major safety challenges in putting patients with life-threatening hypoxemia in the prone position, including disconnection or removal of endotracheal tubes or intravascular catheters, and kinking or secretion-induced plugging of endotracheal tubes. ${ }^{13}$

Despite prone positioning's physiologic advantages, individual randomized controlled trials have not demonstrated its superior clinical outcomes compared with supine positioning. Consequently, we conducted a systematic review and meta-analysis to evaluate the effect of prone positioning on clinical outcomes, including mortality, oxygenation, ventilatorassociated pneumonia, duration of ventilation and adverse events, in patients with acute hypoxemic respiratory failure.

\section{Methods}

\section{Literature search}

We searched MEDLINE, EMBASE, the Cochrane Central Register of Controlled Trials and Science Citation Index Expanded for articles published from database inception to February 2008. Our search strategy is outlined in Appendix I, available at www.cmaj.ca/cgi/content/full/178/9/II53/DC2. We also searched supplementary data sources, including the "related articles" feature on PubMed; bibliographies of included studies and review articles; conference proceedings of the American Thoracic Society (1994-2007), the American College of Chest Physicians (1994-2007), the European Society of Intensive Care Medicine (1994-2007) and the Society of Critical Care Medicine (1994-2008); and clinical trial registries (www .clinicaltrials.gov, www.controlled-trials.com). We contacted clinical experts and the authors of all included studies for additional data. We did not impose language restrictions.

\section{Study selection}

We included studies that met 3 criteria. First, they enrolled adult or pediatric patients with acute hypoxemic respiratory failure (defined as the ratio of partial pressure of oxygen to inspired fraction of oxygen $\leq 300 \mathrm{~mm} \mathrm{Hg}$ ), including acute lung injury and acute respiratory distress syndrome, who received mechanical ventilation. Second, they randomly assigned patients to 2 or more groups, including a treatment group that received ventilation at least once in the prone position and a control group that received ventilation in the supine position. Third, they reported all-cause mortality, the ratio of partial pressure of oxygen to inspired fraction of oxygen, ventilator-associated pneumonia, the duration of ventilation, the number of ventilator-free days from randomization to day 28 or 30 , or adverse events, including pressure ulcers, endotracheal tube obstruction, unplanned extubation, dislodgement of central venous catheters or thoracostomy tubes, pneumothoraces and cardiac arrests. Our quantitative analyses included trials that enrolled adults or postneonatal children. Excluding the pediatric trials did not change any results; therefore, we present only the combined results.

We also considered trials that assigned patients in alternating fashion or by hospital registry number (quasirandomization), or involved cointerventions, such as highfrequency oscillation or nitric oxide, that were specified as part of the intervention and were applied equally to both groups. We used authors' definitions of acute lung injury and acute respiratory distress syndrome. We excluded randomized crossover trials that assigned patients to both treatment and control groups.

\section{Data abstraction and validity assessment}

Each of us independently evaluated studies for inclusion and abstracted data on study methods and outcomes; disagreements were resolved by consensus. Measures of study quality included method and concealment of allocation (adequate $\mathrm{v}$. inadequate), postallocation withdrawals (yes v. no), patients with missing mortality status owing to loss to follow-up (yes v. no), crossovers between groups (yes v. no), analysis of data by group to which patients were originally assigned (yes v. no), blinding of outcome assessors for ventilator-associated pneumonia (blinded or centrally adjudicating assessors $\mathrm{v}$. neither), cointerventions (standardization or equal application of mechanical ventilation, ventilator weaning, sedation and paralysis, and alternative treatments for hypoxemia), and early stopping of the trial before planned enrolment was completed (yes v. no).

We contacted authors of all included trials to clarify methodology and request data missing from prespecified analyses.

\section{Statistical analysis}

The primary outcome was all-cause mortality in the ICU at any time after randomization; if ICU mortality was not reported, we used mortality at 28 or 30 days after randomization or hospital mortality. A priori, we planned subgroup analyses based on patient population (acute lung injury or acute respiratory distress syndrome v. other) and duration of prone positioning (prolonged, which we defined as up to 24 hours daily for more than 2 days, v. short-term).

Secondary outcomes included ventilator-associated pneumonia, the number of days on mechanical ventilation and ventilator-free days, oxygenation on days $\mathrm{I}-3$ and adverse events. Oxygenation outcomes are presented only for days I-3 because the extent of missing data for subsequent days (in trials reporting these outcomes) limits the interpretability of these analyses. To show the maximal effect of prone positioning on oxygenation, we compared the mean ratio of partial pressure of oxygen to inspired fraction of oxygen, measured in the prone group at the end of a prone manoeuvre, 
with the simultaneously recorded measurement in the supine group for each day. For this measurement, day I refers to the end of the first proning session (for sessions lasting less than 24 hours) or the end of 24 hours (for continuous proning lasting longer than 24 hours). To measure the difference in oxygenation that remained after patients in the prone group were returned to the supine position, we compared the mean ratios of partial pressure of oxygen to inspired fraction of oxygen between the prone and supine groups that were measured in the morning (just before the subsequent proning manoeuvre in the prone group). Day I for this measurement refers to the measurement taken the morning after the first proning session and applies only to proning sessions that lasted less than 24 hours. Finally, in a post hoc analysis we compared the mean ratio of partial pressure of oxygen to inspired fraction of oxygen measured within I hour of patients being turned to the prone position with the nearsimultaneous measurement in the supine group.

In our meta-analysis, all statistical tests were 2 -sided, and we considered $p<0.05$ to be statistically significant. We report continuous outcomes as weighted mean differences (a measure of absolute change) for number of days of mechanical ventilation and ventilator-free days, and as ratios of means (a measure of relative change $)^{14}$ for ratio of partial pressure of oxygen to inspired fraction of oxygen. We report binary outcomes (mortality, ventilator-associated pneumonia and adverse events) as risk ratios (RRs). All outcomes are presented with $95 \%$ confidence intervals (CIs). At least 2 of us independently conducted each analysis to minimize data management errors. ${ }^{15}$

We measured heterogeneity and expressed it as $I^{2}$, the percentage of total variation across studies owing to betweenstudy heterogeneity rather than chance, ${ }^{16,17}$ with suggested thresholds for low $\left(I^{2}=25 \%-49 \%\right)$, moderate $\left(I^{2}=\right.$ $50 \%-74 \%)$ and high $\left(I^{2} \geq 75 \%\right)$ values. ${ }^{17}$ A priori hypotheses to explain moderate to high heterogeneity in mortality and ventilator-associated pneumonia included study population and duration of prone positioning, and study quality (adequate $\mathrm{v}$. inadequate concealment of patient assignment, including quasi-randomization, and blinded or centrally adjudicating assessors v. neither [for ventilator-associated pneumonia only]). We performed meta-analyses using a random-effects model, which incorporates within- and between-study variation and provides more conservative treatment estimates when heterogeneity is present. ${ }^{18}$

To assess publication bias, we visually examined the funnel plot for mortality (standard error of the natural logarithm of RR v. RR for each trial) and performed a Begg rank correlation test ${ }^{19}$ and Macaskill regression test, ${ }^{20}$ as modified by Peters and colleagues, ${ }^{21}$ in which we considered $p<0$.Io to be statistically significant.

\section{Results}

\section{Literature search}

Through the searches of bibliographic databases we identified 1676 citations. Of these citations and additional citations retrieved from other sources, we retrieved 50 studies for detailed evaluation and excluded 29 . We selected 13 primary

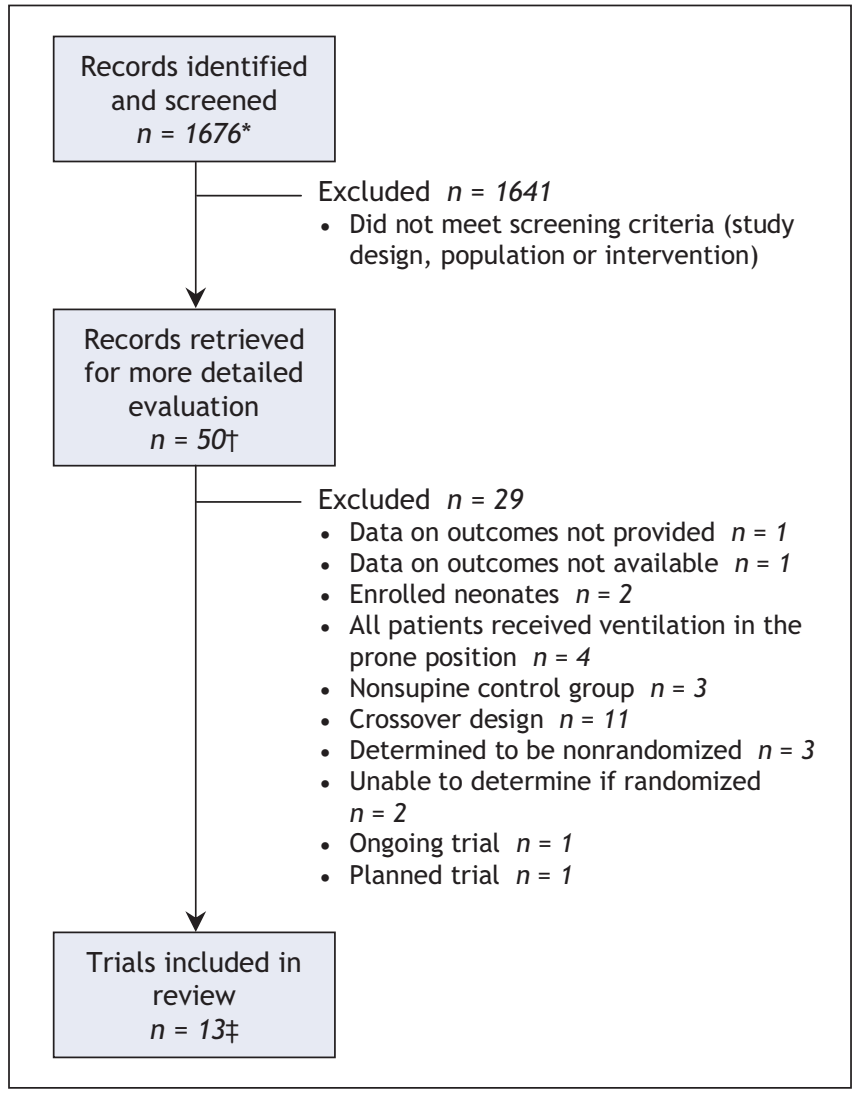

Figure 1: Flow of studies in the systematic review. ${ }^{*}$ Records were identified in electronic database search. †The records retrieved for more detailed evaluation came from the electronic databases and other sources. $\neq$ We included 13 primary trials and 8 references with duplicate or additional data. Of the studies retained for analysis, 12 trials contributed oxygenation data, 10 contributed data on adverse events, and 10 were included in our primary mortality analysis.

randomized and quasi-randomized trials for inclusion in our review and meta-analysis, ${ }^{22-34}$ along with 8 citations providing duplicate or supplementary data (data from 4 of $\mathrm{I}_{3}$ primary trials were distributed among several additional publications) $^{35-42}$ (Figure I). Reviewers had perfect agreement on study inclusion.

The authors of the included trials provided additional clinical $^{22,24,28,30,34}$ and physiologic ${ }^{23,24,28-30,32,34}$ data or clarified data or methods. ${ }^{22-25,28-34}$ The author of 2 of the trials ${ }^{26,27}$ could not provide any additional information.

\section{Study characteristics and methodologic quality}

Appendix 2 (available at www.cmaj.ca/cgi/content/full/178/9 /II53/DC2) describes the I3 included trials, ${ }^{22-34}$ which enrolled a total of 1559 patients (median per trial 28, range I6-802) with acute lung injury, ${ }^{23,26,28,30}$ acute respiratory distress syndrome, ${ }^{22,29,31,32,34}$ or acute hypoxemic respiratory failure. ${ }^{25,27,33}$ The largest trial $(n=802)$ enrolled patients with acute hypoxemic respiratory failure; $51 \%$ had acute lung injury or acute respiratory distress syndrome and $7 \%$ had cardiogenic pulmonary edema. ${ }^{27}$ Most of the trials enrolled patients within 
48 hours of diagnosis. One trial enrolled comatose patients (Glasgow coma score $\leq 9$ ), who were not necessarily in acute hypoxemic respiratory failure, within 24 hours of mechanical ventilation. ${ }^{24}$ Only data for subgroups of patients with hypoxemia (ratio of partial pressure of oxygen to inspired fraction of oxygen $\leq 300 \mathrm{~mm} \mathrm{Hg}$ ), or acute lung injury or acute respiratory distress syndrome were used in our analyses. Two trials enrolled only children. ${ }^{28,33}$

Patients received mechanical ventilation in the prone position for a median of 12 hours per day (range 4-24 hours), and proning manoeuvres continued either for a prespecified per$\operatorname{iod}^{22,25,26,29,32,33}$ or until prespecified clinical improvements occurred $^{23,24,27,28,30,31,34}$ (median duration of proning 4 days, range I-Io days). About 2-6 clinical personnel ${ }^{23,25,28,31}$ were required for each turning procedure (4-6 personnel were required in trials enrolling adults)..$^{23,25,31}$ The turning procedure lasted a mean of Io (standard deviation I2) minutes in the only trial that reported this information. ${ }^{23}$

All but I trial, ${ }^{22}$ which was available only in abstract form, provided some description of mechanical ventilation. Five trials mandated low tidal volume ventilation $(6-8 \mathrm{~mL} / \mathrm{kg}$ body weight), ${ }^{26,28,30,32,34} 4$ trials used protocols to adjust positive endexpiratory pressure, ${ }^{26,28,32,34}$ and 5 trials reported a mean positive end-expiratory pressure of $7-\mathrm{I} 2 \mathrm{~cm} \mathrm{H}_{2} \mathrm{O}$ during the study period. ${ }^{23,27,28,30,31}$ Additional cointerventions that were specified as part of the treatment and applied to all patients in both groups included use of nitric oxide ${ }^{33}$ and high-frequency oscillation. ${ }^{29}$

The trials had high methodologic quality (Appendix 2). Most described adequate allocation concealment (9 trials). ${ }^{22-24,27-32} \mathrm{Al}-$ location was not concealed in 3 trials, ${ }^{25,33,34}$ of which 2 assigned patients using alternate allocation. ${ }^{25,33}$ Nine trials standardized or described at least I other cointervention such as sedation, ${ }^{28,30,31}$ paralysis, ${ }^{29-32}$ or ventilator weaning. ${ }^{24,25,27,28,31,33}$ Four trials reported postrandomization withdrawals: less than $5 \%$ of enrolled patients in 3 trials $\left(9 / 802,{ }^{27} \mathrm{I} / \mathrm{IO} 2,{ }^{28} 6 / \mathrm{I}_{4} 2^{31}\right)$ and $8 \%$ in I trial ${ }^{33}(2 / 24)$. Only I trial ${ }^{27}$ reported any losses to follow-up $(<0.25 \%$ of patients or $2 / 802)$. However, only 2 of the 5 trials reporting ventilator-associated pneumonia ${ }^{24,25,27,30,31}$ partially blinded outcome assessors to treatment group ${ }^{24}$ or adjudicated the outcome, ${ }^{27}$ and only 3 trials provided specific diagnostic criteria for ventilator-associated pneumonia. ${ }^{24,27,30}$ One trial classified pressure ulcers using standardized criteria. ${ }^{23}$ Five trials ${ }^{23,24,27,28,31}$ reported crossovers between groups, which involved less than I0\% of patients in 4 trials (I2/304, $\left.{ }^{23} 2 / 2 \mathrm{I},{ }^{24} 4 / \mathrm{IO} 2,{ }^{28} 5 / \mathrm{I}_{4} 2^{31}\right)$. All trials analyzed data for patients by assigned group. ${ }^{22-34}$ Five trials ended early, I after meeting prespecified futility criteria, ${ }^{28}$ and 4 because of low or declining enrolment. ${ }^{23,24,31,34}$

\section{Clinical outcomes}

\section{Mortality}

In the primary analysis (Io trials, $n=\mathrm{I} 486$ ), ${ }^{22-24,27-32,34}$ ventilation in the prone position had no effect on mortality (RR $0.96,95 \%$ CI 0.84 to I.09; $p=0.52$ ) (Figure 2). Three trials provided no mortality data. ${ }^{25,26,33}$ In the subgroup analysis, we found no significant difference in mortality between trials of short-term prone positioning ${ }^{22,29,32}$ (RR $0.77,95 \% \mathrm{CI} 0.46$ to I.28) and those of prolonged prone positioning $23,24,27,28,30,31,34$ (RR $0.97,95 \%$ CI 0.85 to I.II; $p=0.39$ for comparison of RRs

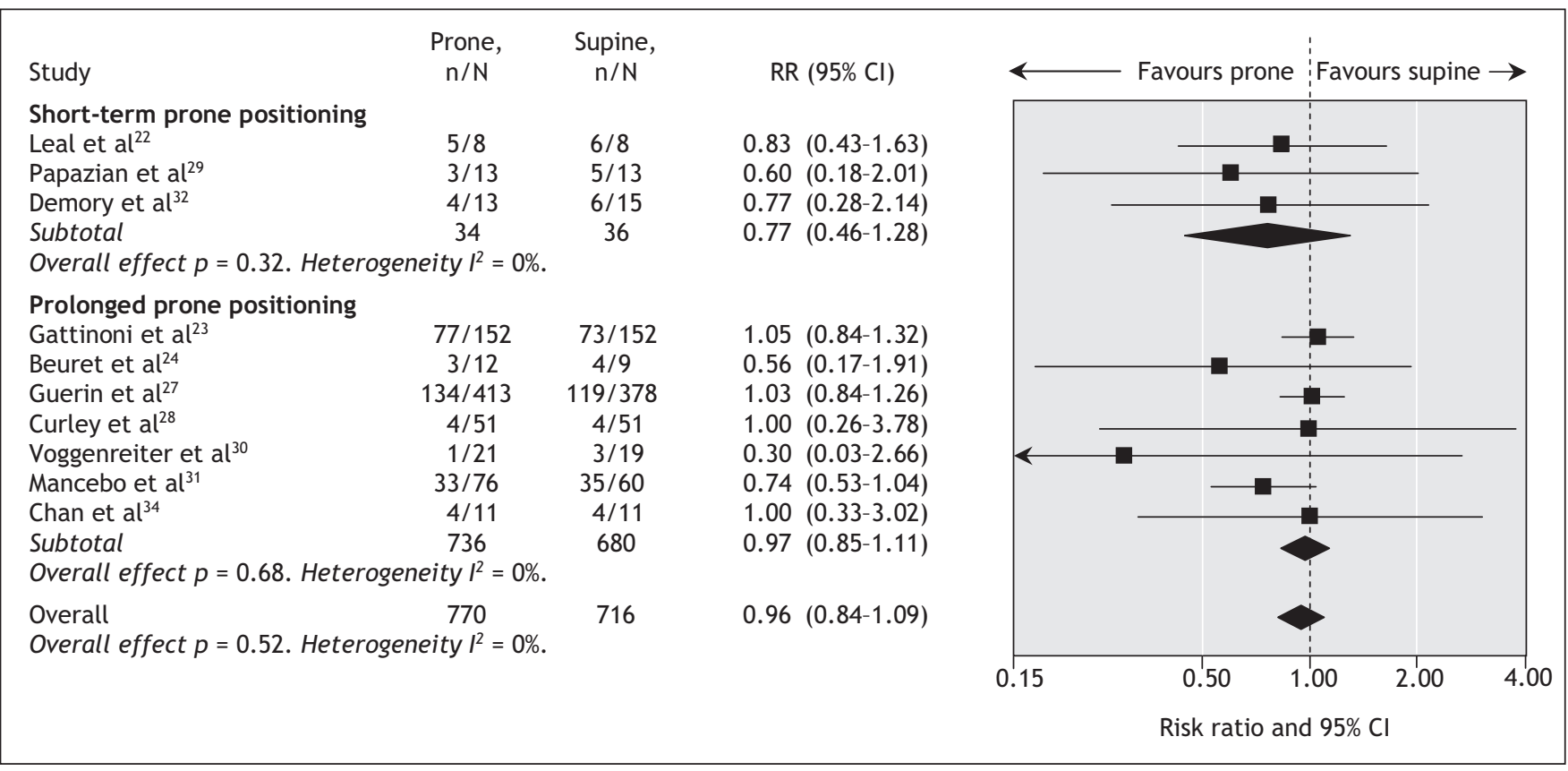

Figure 2: Effect of ventilation in the prone position on mortality. We used a random-effects model in our analysis. The duration of prone positioning was up to 24 hours for 1-2 days in the short-term trials and up to 24 hours daily for more than 2 days in the prolonged-duration trials. One trial ${ }^{24}$ included data only for patients with acute hypoxemic respiratory failure. Including all patients from this trial $(7 / 25$ deaths in the prone group and $14 / 28$ deaths in the supine group) did not change the result ( $\mathrm{RR} 0.95,95 \% \mathrm{Cl} 0.83$ to $1.08 ; p=0.41) . l^{2}=$ percentage of total variation across studies owing to between-study heterogeneity rather than chance. $\mathrm{Cl}=$ confidence interval, $\mathrm{RR}=$ risk ratio. 
using $z$ score) (Figure 2). However, a single trial investigating an extended proning strategy (mean of 17 hours per day for Io days) for acute respiratory distress syndrome showed a trend toward reduced mortality (RR $0.74,95 \% \mathrm{CI} 0.53$ to $1.04 ; p=$ 0.08). ${ }^{31}$ The effect of prone positioning on mortality was also similar (nonsignificant $z$ scores for differences in RRs) in 9 trials $(n=68 \mathrm{I})$ among patients with acute lung injury or acute respiratory distress syndrome $e^{22-24,28-32,34}(\mathrm{RR} 0.92,95 \% \mathrm{CI}$ 0.78 to I.09; $p=0.35$ ) and in 6 trials $(n=6 \mathrm{II})$ in the subset of these patients who had prolonged duration of prone positioning (RR $0.94,95 \% \mathrm{CI} 0.79$ to I.I3; $p=0.53$ )..$^{23,24,28,30,31,34}$ Additional subgroup analyses to explore the effects of study quality and age (children v. adults) were limited because, in each case, one of the subgroup pairs included only a single randomized controlled trial (Appendix 3, available at www .cmaj.ca/cgi/content/full/I78/9/II53/DC2). All mortality analyses showed little to no statistical heterogeneity beyond that expected by chance $\left(I^{2}<10 \%\right)$.

Visual inspection of a funnel plot revealed asymmetry, which suggested under-reporting of smaller trials showing excess mortality in the prone group. However, results of statistical tests did not confirm publication bias (Appendix 4, available at www.cmaj.ca/cgi/content/full/I78/9/II53/DC2).

\section{Oxygenation}

Relative to supine patients, prone ventilation increased the ratio of partial pressure of oxygen to inspired fraction of oxygen by $23 \%-34 \%$ on days I- 3 after randomization, measured at the end of the prone manoeuvre (Table I and Figure 3). Post-hoc analysis revealed that most of this improvement occurred within I hour of the patients being turned to the prone position. The ratio of partial pressure of oxygen to inspired fraction of oxygen remained $6 \%-9 \%$ higher in patients in the prone group after they were returned to the supine position after a prone manoeuvre (Table I) (Appendix 5, available at www.cmaj.ca/cgi/content/full/178/9/1153/DC2).

\section{Ventilator-associated pneumonia and duration of ventilation}

In 6 trials $(n=\mathrm{I0} 26),{ }^{24,25,27,30,31,34}$ ventilation in the prone position reduced the risk of ventilator-associated pneumonia (RR 0.8I, $95 \%$ CI 0.66 to $0.99, p=0.04)$, with no statistical heterogeneity beyond that expected by chance $\left(I^{2}=0 \%\right)$ (Figure 4). One of the 6 trials $(n=22)$ recorded no cases of ventilator-associated pneumonia. ${ }^{34}$ The effect was similar in trials that blinded ventilator-associated pneumonia assessors $^{24}$ or adjudicated the outcome ${ }^{27}$ ( $p=0.89$ for comparison of RRs in these v. other trials). Although in 6 trials $(n=992)^{24,25,27-30}$ patients who received ventilation in the prone position generally had shorter durations of ventilation (weighted mean difference -0.9 days, $95 \% \mathrm{CI}-$ I.9 to o.I; $p=$ $\left.0.06, I^{2}=3 \%\right)$, in 4 trials $(n=148),{ }^{23,24,28,29}$ the number of ventilator-free days in the prone group was not significantly greater than the number in the supine group (weighted mean difference 3.7 days, $95 \%$ CI -I.8 to $9.3 ; p=0.19, I^{2}=67 \%$ ).

\section{Adverse events}

In 6 trials $(n=504),{ }^{22-24,28,30,34}$ ventilation in the prone position increased the risk of pressure ulcers (RR I. $36,95 \% \mathrm{CI}$ I.07 to I.7I; $p=0.0$ I, $P=0 \%$ ). Between 5 and 8 trials contributed data to analyses of other adverse events; these analyses showed no increased risks (Figure 5, Table 2). When data

Table 1: Effect of ventilation in the prone position on oxygenation, by timing of measurement of ratio of partial pressure of oxygen to inspired fraction of oxygen*

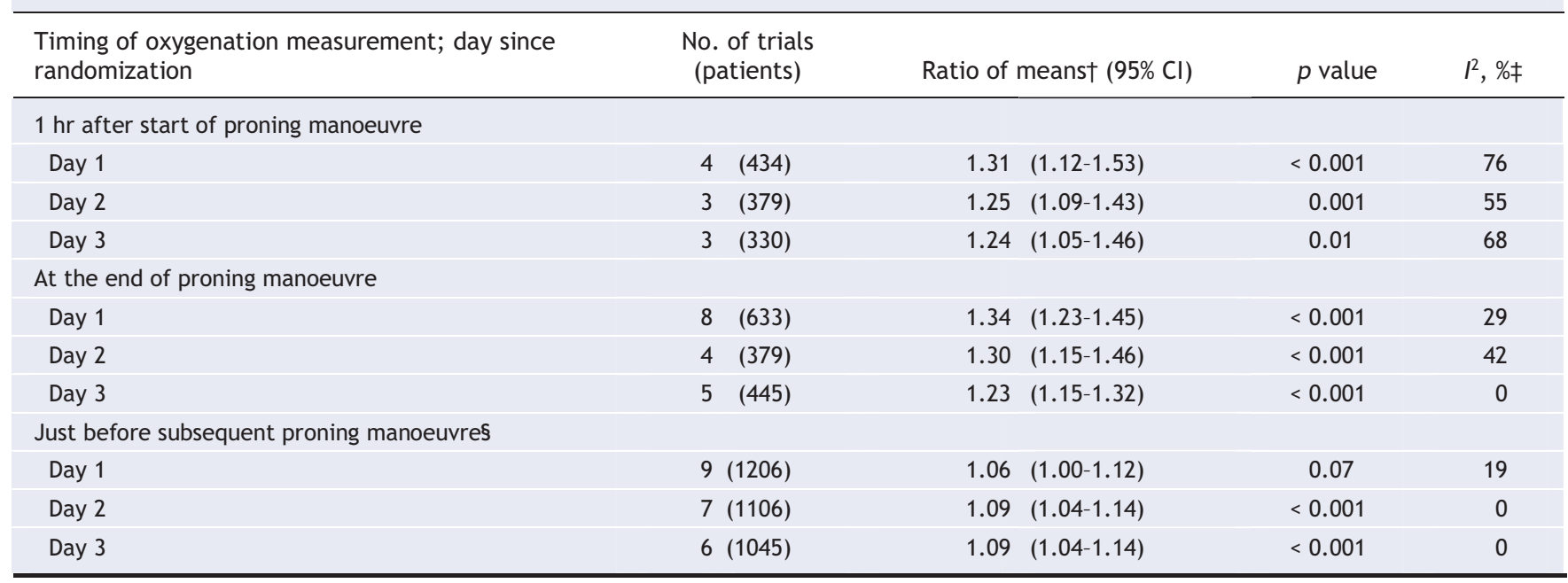

Note: $\mathrm{Cl}=$ confidence interval.

*The author of 1 trial $^{33}$ confirmed that the published error terms for ratios of partial pressure of oxygen to inspired fraction of oxygen were standard deviations rather than standard errors of means. Meta-analyses of oxygenation index (defined as $100 \times$ mean airway pressure/ratio of partial pressure of oxygen to inspired fraction of oxygen) are not shown because only 3 trials ${ }^{28,29,33}$ reported these data at any time, with only 1 trial ${ }^{28}$ providing data beyond day 1 . These 3 trials all reported the ratio of partial pressure of oxygen to inspired fraction of oxygen and oxygenation index simultaneously.

†Ratio of means is the mean ratio of partial pressure of oxygen to inspired fraction of oxygen in the prone group divided by that in the control group. Random-effects models were used in all analyses.

$\neq l^{2}=$ percentage of total variation across studies owing to between-study heterogeneity rather than chance.

$\S$ Data were recorded in the morning, just before the subsequent proning manoeuvre, when patients in the prone group were in the supine position. On day 1 , patients in the prone group had already completed 1 manoeuvre. 
from one trial ${ }^{27}$ reporting the number of occurrences of adverse events instead of the number of patients who experienced adverse events per group were included, the risk of endotracheal tube obstruction became statistically significant (RR 2.46, 95\% CI I.33 to $4.55 ; p=0.004, P=0 \%$ ). ${ }^{27-29}$

\section{Interpretation}

Our systematic review suggests that mechanical ventilation in the prone position does not improve survival for patients with acute hypoxemic respiratory failure, including acute lung injury and acute respiratory distress syndrome, despite improved oxygenation and a reduced risk of ventilator-associated pneumonia. Prone positioning increased the risk of pressure ulcers and possibly endotracheal tube obstruction, but otherwise it was safe. Despite variable duration of ventilation in the prone position and clinically diverse populations in the included trials, pooled clinical outcomes had little statistical heterogeneity, which strengthens our findings. These results do not justify the routine use of prone positioning during mechanical ventilation in patients with acute hypoxemic respiratory failure.

There are several hypotheses that may explain the neutral effect of ventilation in the prone position on mortality. First, short- to medium-term improved oxygenation may not increase survival ${ }^{43}$ because of poor correlation between oxygenation and severity of lung injury. Indeed, patients with acute respiratory distress syndrome die more often of multiple organ failure than hypoxemia. ${ }^{44,45}$ Although prone positioning improves oxygenation within I hour and to a greater extent than inhaled nitric oxide, ${ }^{3}$ such improvements may help only the most severely hypoxemic patients to survive. Gattinoni and colleagues $^{23}$ reported a post hoc analysis showing a significantly lower Io-day mortality rate with prone positioning in the subgroup of patients with the lowest ratio of partial pressure of oxygen to inspired fraction of oxygen. In contrast, Mancebo and colleagues ${ }^{31}$ did not find the initial ratio of partial pressure of oxygen to inspired fraction of oxygen to be a significant predictor of mortality in a multivariable logistic regression model. Neither study reported a statistical test of the interaction between treatment group and initial ratio of partial pressure of oxygen to inspired fraction of oxygen. No randomized controlled trials have investigated prone positioning during ventilation as rescue therapy for critical hypoxemia. Prone positioning in such patients may prevent imminent death and allow time for other treatments to help.

Second, the broad nature of selection criteria in the in-

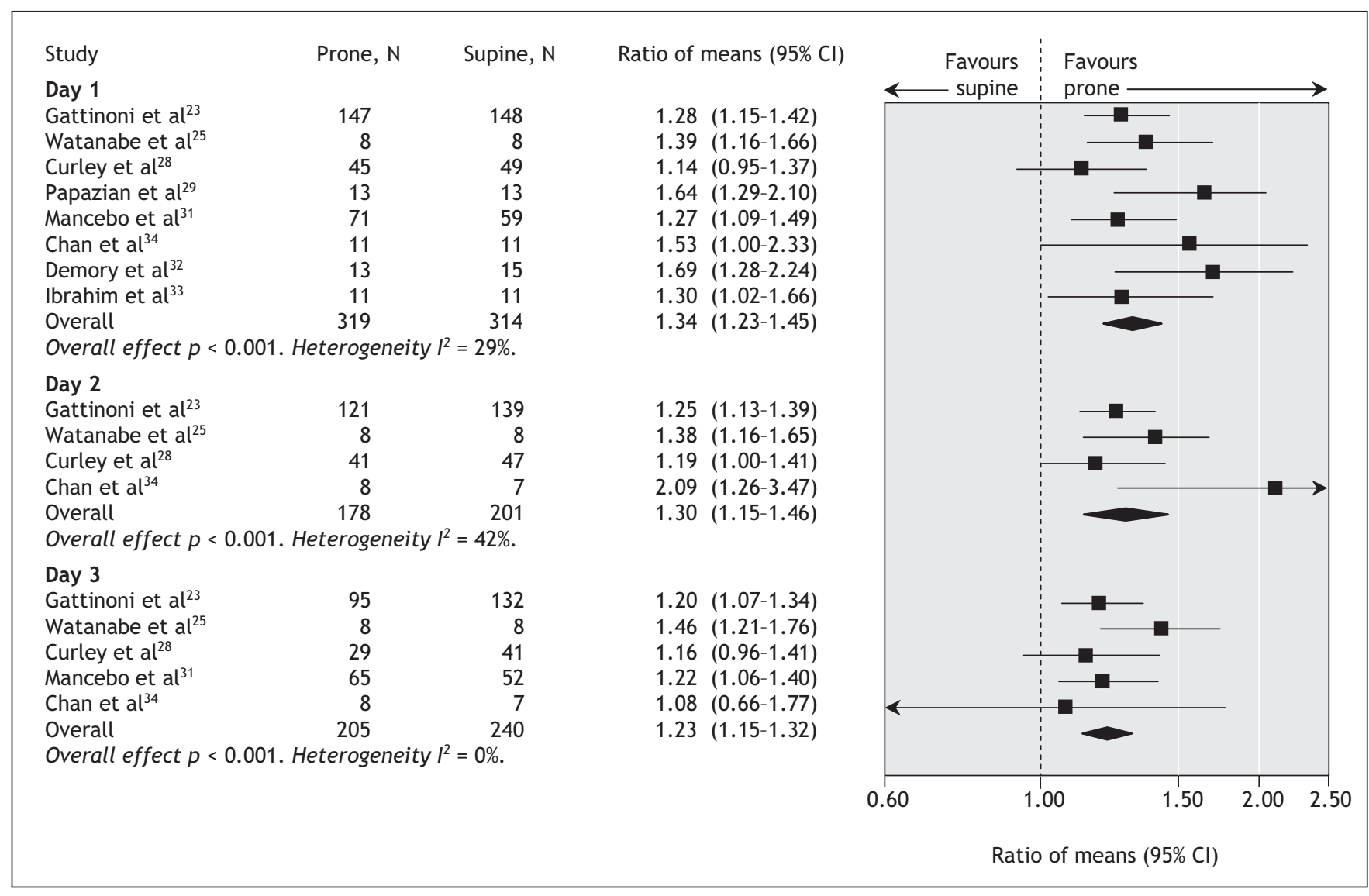

Figure 3: Effect of ventilation in the prone position on daily ratio of partial pressure of oxygen to inspired fraction of oxygen. We used a random-effects model in our analysis. Values were recorded at the end of the period of prone positioning (prone group) and simultaneously in the supine group. Ratio of means = mean ratio of partial pressure of oxygen to inspired fraction of oxygen in the prone group divided by that in the supine group. $P^{2}$ = percentage of total variation across studies owing to between-study heterogeneity rather than chance. $\mathrm{Cl}=$ confidence interval. 
cluded trials may have failed to identify a particular population that would benefit from ventilation in the prone position. Several trials enrolled patients with diverse types of respiratory failure. ${ }^{24,25,27,30}$ In the largest trial, only $5 \mathrm{I} \%$ of the 802 patients had acute lung injury or acute respiratory distress syndrome. ${ }^{27}$ Although meta-analysis restricted to patients with acute lung injury or acute respiratory distress syndrome did not show a mortality benefit, physiologic variables (other than oxygenation response) may identify a subgroup of these patients who might benefit from ventilation in the prone position. For example, Gattinoni and colleagues ${ }^{36}$ reported in a post hoc analysis that decreased partial pressure of carbon dioxide after an initial 6-hour prone period, which likely reflected a lower fraction of minute ventilation delivered to nonperfused lungs (dead space), was associated with improved survival.

Third, the duration of prone positioning may have been insufficient. Our subgroup analysis did not show benefit among all prolonged-duration trials; however, the most intensive proning regimen studied (I7 hours daily for Io days) was shown in an adjusted analysis to reduce mortality. ${ }^{31}$ An ongoing randomized controlled trial of mechanical ventilation with an intensive proning regimen (20 hours daily for up to 28 days) involving 340 patients with acute respiratory distress syndrome (the Prone-Supine Study II [www.clinicaltrials.gov /ct2/show/NCToor59939?term=NCToor59939\&rank=I]) may provide valuable additional data regarding this issue.

Finally, it is possible that the benefits of ventilation in the prone position were overshadowed by a mechanical ventilation strategy that injured the lungs and perpetuated multiple organ failure. The ongoing Prone-Supine Study II mandates lung-protective mechanical ventilation, which may resolve this issue. Another planned randomized controlled trial ${ }^{46}$ would add to current knowledge by enrolling patients with severe acute respiratory distress syndrome (ratio of partial pressure of oxygen to inspired fraction of oxygen no greater than $100 \mathrm{~mm} \mathrm{Hg}$ and high severity of illness) and mandating prolonged prone positioning and lung-protective ventilation.

Our meta-analysis demonstrated a reduced risk of ventilator-associated pneumonia associated with prone positioning. Some small nonrandomized studies suggested better drainage of respiratory secretions with this technique, ${ }^{9,10}$ which may prevent aspiration. However, our finding is limited by potential ascertainment bias because most of the trials lacked standard diagnostic criteria and blinding of outcomes assessors. Furthermore, the clinical importance of reduced ventilator-associated pneumonia with prone positioning is

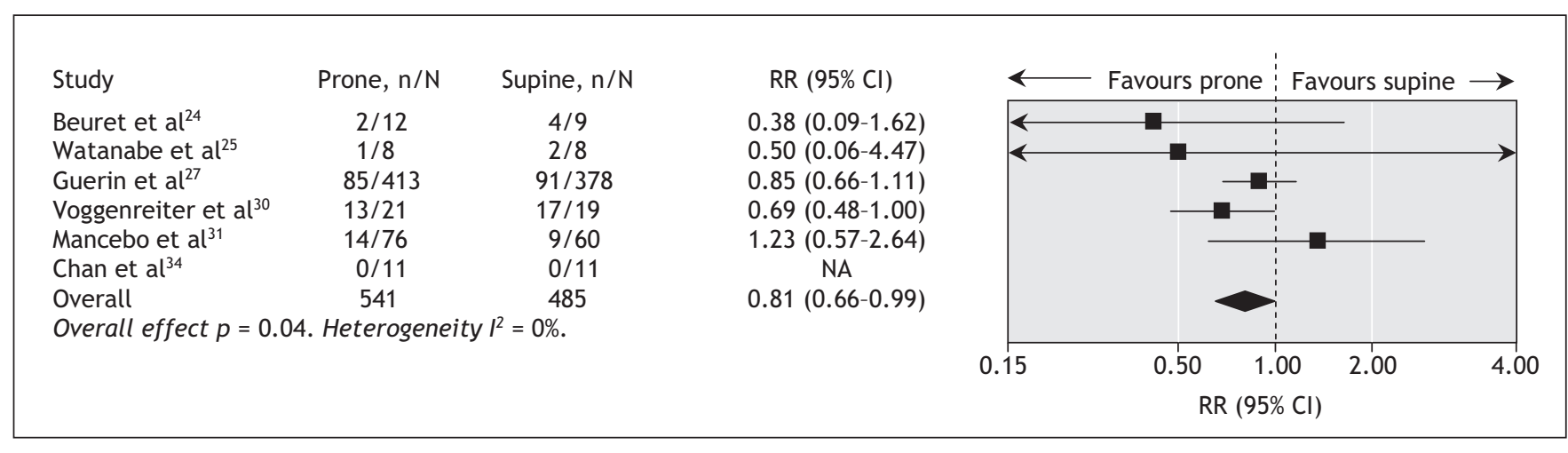

Figure 4: Effect of ventilation in the prone position on risk of ventilator-associated pneumonia. We used a random-effects model in our analysis. One trial ${ }^{24}$ included data only for patients with acute hypoxemic respiratory failure. $l^{2}=$ percentage of total variation across studies owing to between-study heterogeneity rather than chance. $\mathrm{Cl}=$ confidence interval, $\mathrm{NA}=$ not applicable, $\mathrm{RR}=$ risk ratio.

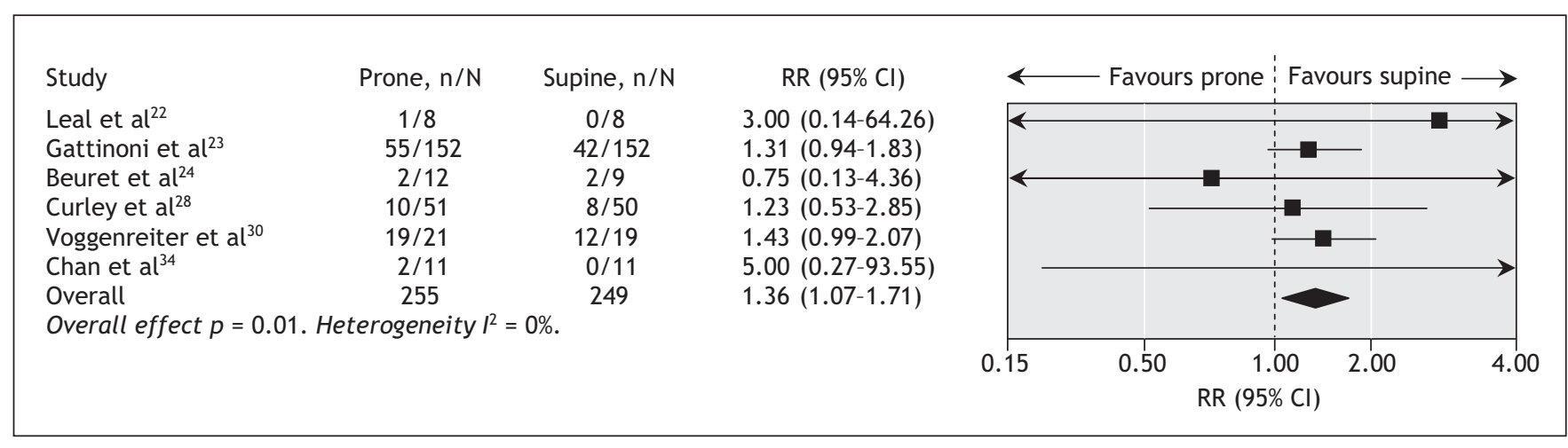

Figure 5: Effect of ventilation in the prone position on risk of pressure ulcers. We used a random-effects model in our analysis. One trial ${ }^{24}$ included data only for patients with acute hypoxemic respiratory failure. $l^{2}=$ percentage of total variation across studies owing to between-study heterogeneity rather than chance. $\mathrm{Cl}=$ confidence interval, $\mathrm{RR}=$ risk ratio. 
unclear given similar duration of ventilation, ventilator-free days and mortality among patients who received ventilation in the prone and supine positions.

We found mechanical ventilation in the prone position to be generally safe, at least in centres participating in clinical trials. However, the procedure was labour-intensive, requiring 4-6 personnel to turn an adult patient, and it increased the risk of pressure ulcers. In contrast, with the exception of possible endotracheal tube blockage, prone positioning did not increase the risk of potentially life-threatening complications such as accidental extubation and dislodgement of central catheters or thorocostomy tubes. Nevertheless, some trials reported airway obstruction, accidental extubation and dislodgement of central catheters directly related to the turning procedure, ${ }^{23,31}$ which led to cardiac arrest in one instance. ${ }^{31}$ Such complications, although infrequent, could be catastrophic in patients with critical hypoxemia. Less experienced centres may face more life-threatening complications, but turning protocols and nursing care guidelines may mitigate the risk. ${ }^{13,38,47}$

A recent survey of 702 (predominantly adult) ICUs in Germany ${ }^{48}$ reported more complications during various forms of positioning therapy than recorded in randomized controlled trials. These included hemodynamic instability (reported by $74 \%$ of ICUs), accidental removal of tubes or catheters $(50 \%)$, worsening gas exchange ( $45 \%)$, patient intolerance owing to inadequate sedation ( $4 \mathrm{r} \%)$ and cardiac arrhythmias $(22 \%)$. Moreover, many respondents believed that successful application of positioning therapy forced clinicians to compromise other aspects of critical care by requiring them to deepen sedation ( $77 \%$ of ICUs) and to stop (I $6 \%$ ) or at least reduce $(33 \%)$ enteral feeds. Another survey of proning practices in 25 ICUs in Belgium reported similar rates of serious complications, in addition to increased workload (owing to increased suctioning and eye care), which contributed to the reluctance of nurses in 9 ICUs ( $36 \%$ ) to use the technique. ${ }^{12}$
Strengths of our review include methods to reduce bias and analysis of a comprehensive set of prespecified clinical and physiologic outcomes. Our study had a number of limitations, including variability in the selection criteria of individual trials (including author definitions of acute lung injury and acute respiratory distress syndrome) and reduced sample size in 5 trials that ended early because of futility or declining enrolment. Both factors may have diluted our ability to detect a survival benefit through meta-analysis. In addition, although the mortality funnel plot suggested publication bias (Appendix 4, available at www.cmaj.ca/cgi/content/full//78/9/II53/DC2), results of statistical tests did not confirm the presence of publication bias. Statistical tests may fail to detect publication bias, but the assumption of such bias in our meta-analysis would imply that small unpublished randomized trials have shown higher rates of mortality in the prone group than the supine group. Data from such trials would only move the estimated pooled RR for mortality closer to no effect. Another limitation is that supplementary information was not available for all trials, 2 of which were published only as abstracts. In addition, the small number of trials included in our review reduced the precision of the pooled estimates for some clinical and physiologic analyses and may have underestimated heterogeneity. Finally, findings from the largest trial $^{27}$ dominated the meta-analysis of ventilator-associated pneumonia and endotracheal tube obstruction.

In summary, our systematic review found that ventilation in the prone position in patients with acute hypoxemic respiratory failure improved oxygenation and reduced the risk of ventilatorassociated pneumonia, but it did not improve survival. The technique appeared safe in expert centres; however, serious airway, catheter and tube complications may occasionally occur, and the technique increased the risk of pressure ulcers. Consequently, we do not recommend the routine use of prone positioning for patients with hypoxemic respiratory failure. Despite the neutral effect on mortality, clinicians may still consider

Table 2: Risk of adverse events in 12 trials of prone positioning for mechanical ventilation included in our systematic review*

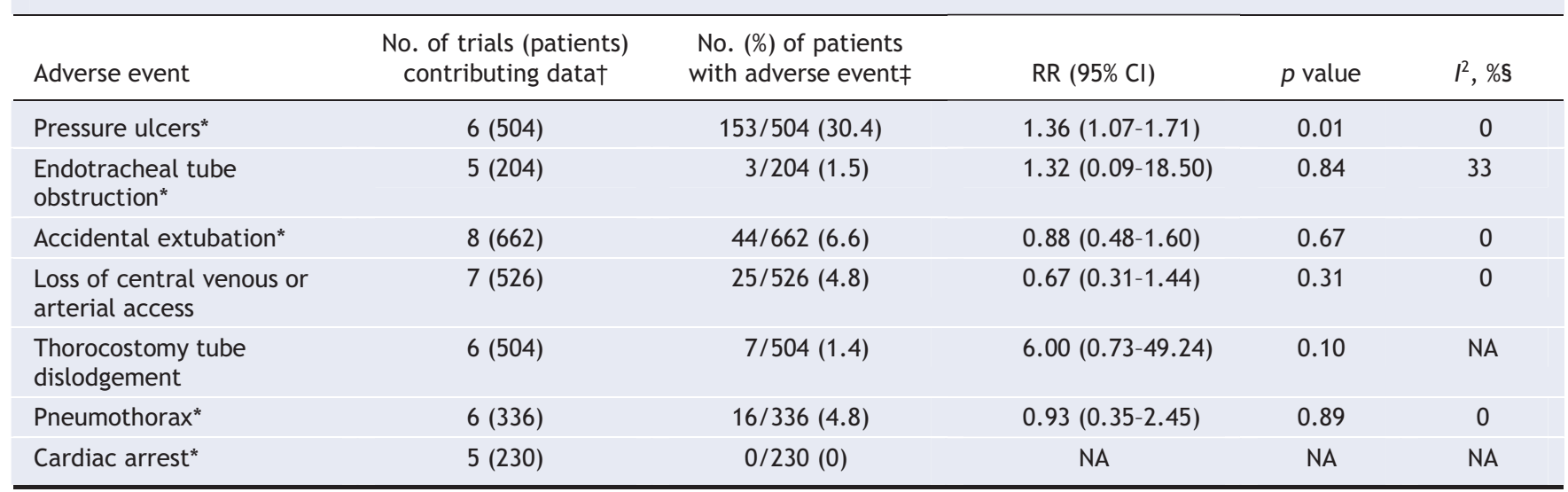

Note: $\mathrm{Cl}=$ confidence interval, $\mathrm{NA}=$ not applicable, $\mathrm{RR}=$ risk ratio. Random-effects models were used in all analyses.

*We excluded the trial by Guerin et $\mathrm{al}^{27}$ from the analysis because it reported the number of occurrences of adverse events rather than the number of patients with adverse events. Assuming a similar distribution of occurrences per patient in the prone and supine groups, when we included these data, the risk of endotracheal tube obstruction became statistically significant (RR $2.46,95 \% \mathrm{Cl} 1.33-4.55 ; p=0.004 ; l^{2}=0 \%$ ) and the risk of pressure ulcers became more significant (RR $1.25,95 \% \mathrm{Cl}$ $\left.1.10-1.43 ; p<0.001 ; l^{2}=0 \%\right)$. This trial contributes heavily to the pooled RRs when its data are included ( $92 \%$ weighting in the endotracheal tube obstruction analysis and $70 \%$ in the pressure ulcer analysis).

tWe counted trials that collected data on the adverse event, regardless of whether an event occurred.

$\ddagger$ This is the unweighted proportion of patients in the prone and supine groups (in trials collecting data on adverse events) who experienced an adverse event.

$\S /^{2}=$ percentage of total variation across studies owing to between-study heterogeneity rather than chance. 
prone positioning for life-threatening hypoxemia, along with other supportive therapies. Current data for such patients are limited, and early termination of several published trials owing to slow enrolment suggests that additional studies, although highly desirable, will be challenging to complete.

\section{This article has been peer reviewed.}

Competing interests: None declared.

Contributors: Sachin Sud, Jan Friedrich and Neill Adhikari conceived and designed the study; acquired, analyzed and interpreted data; and drafted the manuscript. Maneesh Sud acquired data. All of the authors revised the manuscript for important intellectual content and approved the final version. Jan Friedrich and Neill Adhikari provided administrative support and contributed equally to this study.

Acknowledgements: We thank all authors who provided additional data on their trials included in the review: Pascal Beuret; Martha A.Q. Curley; Hala ElMohamady; Paolo Taccone and Luciano Gattinoni; Guillermo DominguezCherit; Jordi Mancebo; Laurent Papazian; Alexey Vlasenko; Greggor Voggenreiter; Ippei Watanabe and Hideyoshi Fujihara; and Chieh-Liang Wu and Ming-Chen Chan. We also thank Ruxandra Pinto for verification of the publication bias analyses and assistance with figures, Art Slutsky for facilitating contact with some of the trial authors, Alex Briskin for translation of Russian papers, and Elizabeth Uleryk for assistance developing the search strategy.

This study received no specific funding. Jan Friedrich is a clinicianscientist of the Canadian Institutes of Health Research.

\section{REFERENCES}

I. Bernard GR, Artigas A, Brigham KL, et al. The American-European Consensus Conference on ARDS. Definitions, mechanisms, relevant outcomes, and clinical trial coordination. Am J Respir Crit Care Med i994;149:818-24.

2. Artigas A, Bernard GR, Carlet J, et al. The American-European Consensus Conference on ARDS, part 2: Ventilatory, pharmacologic, supportive therapy, study design strategies, and issues related to recovery and remodeling. Acute respiratory distress syndrome. Am J Respir Crit Care Med I998;157:1332-47.

3. Adhikari NK, Burns KE, Friedrich JO, et al. Effect of nitric oxide on oxygenation and mortality in acute lung injury: systematic review and meta-analysis. BMJ 2007;334:779.

4. Derdak S, Mehta S, Stewart TE, et al. High-frequency oscillatory ventilation for acute respiratory distress syndrome in adults: a randomized, controlled trial. Am Respir Crit Care Med 2002; 166:801-8.

5. Bollen CW, van Well GTh, Sherry T, et al. High frequency oscillatory ventilation compared with conventional mechanical ventilation in adult respiratory distress syndrome: a randomized controlled trial [ISRCTN24242669]. Crit Care 2005;9:R430-9.

6. Ferguson ND, Chiche JD, Kacmarek RM, et al. Combining high-frequency oscillatory ventilation and recruitment maneuvers in adults with early acute respiratory distress syndrome: the Treatment with Oscillation and an Open Lung Strategy (TOOLS) trial pilot study. Crit Care Med 2005;33:479-86.

7. Bryan AC. Conference on the scientific basis of respiratory therapy. Pulmonary physiotherapy in the pediatric age group. Comments of a devil's advocate. Am Rev Respir Dis 1974;110:143-4.

8. Pelosi P, Brazzi L, Gattinoni L. Prone position in acute respiratory distress syndrome. Eur Respir J 2002;20:1017-28.

9. Boiteau R, Reignier J, Tenaillon A. Prone position consequences on tracheal secretions [abstract]. Am J Respir Crit Care Med I997;155:A89.

Io. Gillart T, Bazin JE, Guelon D, et al. Effect of bronchial drainage on the improvement in gas exchange observed in ventral decubitus in ARDS [article in French]. Ann Fr Anesth Reanim 2000;19:156-63.

II. Gattinoni L, Carlesso E, Cadringher P, et al. Physical and biological triggers of ventilator-induced lung injury and its prevention. Eur Respir J Suppl 2003;47:15s-25s.

I2. Leonet S, Fontaine C, Moraine JJ, et al. Prone positioning in acute respiratory failure: survey of Belgian ICU nurses. Intensive Care Med 2002;28:576-80.

13. Messerole E, Peine P, Wittkopp S, et al. The pragmatics of prone positioning. Am J Respir Crit Care Med 2002;165:1359-63.

I4. Friedrich JO, Adhikari N, Herridge MS, et al. Meta-analysis: low-dose dopamine increases urine output but does not prevent renal dysfunction or death. Ann Intern Med 2005;142:510-24.

I5. Gotzsche PC, Hrobjartsson A, Maric K, et al. Data extraction errors in metaanalyses that use standardized mean differences. JAMA 2007;298:430-7.

I6. Higgins JP, Thompson SG. Quantifying heterogeneity in a meta-analysis. Stat Med 2002;2I:1539-58.

I7. Higgins JP, Thompson SG, Deeks JJ, et al. Measuring inconsistency in metaanalyses. BMJ 2003;327:557-6o.

18. DerSimonian R, Laird N. Meta-analysis in clinical trials. Control Clin Trials I986;7:177-88.
I9. Begg CB, Mazumdar M. Operating characteristics of a rank correlation test for publication bias. Biometrics I994;50:I088-I0I.

20. Macaskill P, Walter SD, Irwig L. A comparison of methods to detect publication bias in meta-analysis. Stat Med 200I;20:64I-54.

2I. Peters JL, Sutton AJ, Jones DR, et al. Comparison of two methods to detect publication bias in meta-analysis. JAMA 2006;295:676-80.

22. Leal RP, Gonzalez R, Gaona C, et al. Randomized trial compare prone vs supine position in patients with ARDS [abstract]. Am J Respir Crit Care Med I997;155:A745.

23. Gattinoni L, Tognoni G, Pesenti A, et al. Effect of prone positioning on the survival of patients with acute respiratory failure. N Engl J Med 200I;345:568-73.

24. Beuret $\mathrm{P}$, Carton MJ, Nourdine K, et al. Prone position as prevention of lung injury in comatose patients: a prospective, randomized, controlled study. Intensive Care Med 2002;28:564-9.

25. Watanabe I, Fujihara H, Sato K, et al. Beneficial effect of a prone position for patients with hypoxemia after transthoracic esophagectomy. Crit Care Med 2002;30:1799-802.

26. Gaillard S, Couder P, Urrea V, et al. Prone position effects on alveolar recruitment and arterial oxygenation in acute lung injury [abstract]. Intensive Care Med 2003;29:SI2.

27. Guerin C, Gaillard S, Lemasson S, et al. Effects of systematic prone positioning in hypoxemic acute respiratory failure: a randomized controlled trial. JAMA 2004;292:2379-87.

28. Curley MA, Hibberd PL, Fineman LD, et al. Effect of prone positioning on clinical outcomes in children with acute lung injury: a randomized controlled trial. JAMA 2005;294:229-37.

29. Papazian L, Gainnier M, Marin V, et al. Comparison of prone positioning and high-frequency oscillatory ventilation in patients with acute respiratory distress syndrome. Crit Care Med 2005;33:2162-7I.

3o. Voggenreiter G, Aufmkolk M, Stiletto RJ, et al. Prone positioning improves oxygenation in post-traumatic lung injury - a prospective randomized trial. J Trauma 2005;59:333-4I.

31. Mancebo J, Fernandez R, Blanch L, et al. A multicenter trial of prolonged prone ventilation in severe acute respiratory distress syndrome. Am J Respir Crit Care Med 2006;173:1233-9.

32. Demory D, Michelet P, Arnal JM, et al. High-frequency oscillatory ventilation following prone positioning prevents a further impairment in oxygenation. Crit Care Med 2007;35:I06-II.

33. Ibrahim TS, El-Mohamady HS. Inhaled nitric oxide and prone position: How far they can improve oxygenation in pediatric patients with acute respiratory distress syndrome? J Med Sci 2007;7:390-5.

34. Chan MC, Hsu JY, Liu HH, et al. Effects of prone position on inflammatory markers in patients with ARDS due to community-acquired pneumonia. $J$ Formos $\mathrm{Med}$ Assoc 2007; I06:708-I6.

35. Gattinoni L, Tognoni G, Brazzi L, et al. Ventilation in the prone position. The Prone-Supine Study Collaborative Group [letter]. Lancet I997;350:815

36. Gattinoni L, Vagginelli F, Carlesso E, et al. Decrease in $\mathrm{PaCO}_{2}$ with prone position is predictive of improved outcome in acute respiratory distress syndrome. Crit Care Med 2003;31:2727-33

37. Aufmkolk M, Voggenreiter G, Mattern T, et al. Effect of prone position on lung surfactant composition and function in multiple trauma patients with respiratory dysfunction. Eur J Trauma 2005;31:33-8.

38. Curley MA, Arnold JH, Thompson JE, et al. Clinical trial design - effect of prone positioning on clinical outcomes in infants and children with acute respiratory distress syndrome. J Crit Care 2006;21:23-32.

39. Fineman LD, LaBrecque MA, Shih MC, et al. Prone positioning can be safely performed in critically ill infants and children. Pediatr Crit Care Med 2006;7:413-22.

40. Lemasson S, Ayzac L, Girard R, et al. Does gas exchange response to prone position predict mortality in hypoxemic acute respiratory failure? Intensive Care Med 2006;32:1987-93.

4I. Friedrich JO, Sud S, Sud M, et al. Prone position ventilation for communityacquired pneumonia [letter]. J Formos Med Assoc 2008;107:I9I.

42. Chan MC, Hsu JY, Liu HH, et al. Reply to Friedrich et al [letter]. J Formos Med Assoc 2008;107:I92.

43. Ventilation with lower tidal volumes as compared with traditional tidal volumes for acute lung injury and the acute respiratory distress syndrome. The Acute Respiratory Distress Syndrome Network. N Engl J Med 2000;342:130I-8.

44. Montgomery AB, Stager MA, Carrico CJ, et al. Causes of mortality in patients with the adult respiratory distress syndrome. Am Rev Respir Dis 1985;132:485-9.

45. Stapleton RD, Wang BM, Hudson LD, et al. Causes and timing of death in patients with ARDS. Chest 2005;128:525-32.

46. Mentzelopoulos SD, Roussos C, Zakynthinos SG. Prone position in early and severe acute respiratory distress syndrome: a design for a definitive randomized controlled trial. Anesth Analg. 2007; 104:466-468.

47. McCormick J, Blackwood B. Nursing the ARDS patient in the prone position: the experience of qualified ICU nurses. Intensive Crit Care Nurs 200I;17:33I-40.

48. Bein T, Ritzka M, Schmidt F, et al. Positioning therapy in intensive care medicine in Germany. Results of a national survey [German]. Anaesthesist 2007;56:226-3I.

Correspondence to: Dr. Jan O. Friedrich, Departments of Critical Care and Medicine, St. Michael's Hospital, 30 Bond St., Bond Wing, Rm. 4-oI5, Toronto ON M5B IW8; fax 4I6 864-6or3; j.friedrich@utoronto.ca 